\title{
Role of multipolarity-Six deformation parameter on exotic decay half-lives of Berkelium nucleus
}

\author{
G. M. Carmel Vigila Bai ${ }^{1}$, R. Nithya Agnes ${ }^{2 *}$ \\ Department of Physics, Rani Anna Government College for women, Tirunelveli - 08, Tamil Nadu, India. \\ Department of Physics, St. John's College, Palayamkottai- 02,Tamil Nadu, India.
}

\begin{abstract}
Using Cubic plus Yukawa plus Exponential Model (CYEM), we have calculated the half lives of various clusters from neutron rich ${ }^{238-252}$ Bk parent nuclei. In our model, the zero point vibration energy is explicitly included without violating the conservation of energy and the nuclear inertia mass coefficient dependent on the centre of mass distance has been used. We have made our calculations by considering Coulomb, Yukawa plus exponential potential as interacting barrier for separated fragments and cubic potential for the overlapping region. The calculated half-lives are compared with other models and are found to be good agreement with each other. In this work, we have examined the changes in half-life value of exotic decay by incorporating hexacontatetrapole $\left(\beta_{6}\right)$ parameter in the parent nucleus along with the quadruple $\left(\beta_{2}\right)$, and hexadecaple $\left(\beta_{4}\right)$ parameters for Berkelium isotopes. These deformations lower the half life value because; it reduces the height and width of the potential barrier. It is also found that the neutron excess in the parent nuclei slows down the cluster decay process. Most of the emitted clusters are alpha like nuclei. The branching ratio relative to alpha has also been calculated.
\end{abstract}

Keywords: Exotic decay, $Q$-value, spontaneous fission and Deformation,

\section{Introduction:}

The spontaneous emission of fragments heavier than alpha particles but lighter than lightest fission fragment observed. Theoretically such emissions were first predicated by Sandulescu, Poenaru and Greiner[1]. The first experimental observation was made by Rose and Jones[2].There exits two kinds of model in explaining the exotic decay process (i) the Pre-formed cluster model and (ii) the fission model. In Pre-formed cluster model [3-5] the cluster is assumed to be pre-born in a parent nucleus before it penetrate the barrier. In fission model [68] the nucleus deforms continuously as it penetrates the nuclear interacting barrier and reaches the scission configuration after running down the coulomb barrier. The role of deformation effect on half lives in cluster decay has been calculated by many authors using different theoretical models [9-12]. Carmel et al [13] have calculated half life for exotic decay in the Trans-Tin region by incorporating deformation effects in parent and daughter or cluster, using cubic plus Yukawa plus exponential (CYE) model. Umai et al [14] have studied the impact of higher multi- polarity parameter $\left(\boldsymbol{\beta}_{6}\right)$ on half-life time of super heavy elements along with the quadrupole, and hexadecapole deformations using CYE model. We have already studied the effects of quadrupole, and hexadecapole deformations of the decaying parent nucleus along with that of emitted cluster and daughter nucleus on half-life of cluster decay for some neutron rich elements in trans-actinide region using CYE model[15-17].In this paper, we have calculated the half of all the possible cluster emission from the neutron rich element of $\mathrm{Bk}$ isotopes by incorporating $\left(\boldsymbol{\beta}_{\mathbf{6}}\right)$ parameter in the parent nucleus along with the ground state $\left(\boldsymbol{\beta}_{\mathbf{2}}\right.$ and, $\left.\boldsymbol{\beta}_{4}\right)$ deformation parameters. We have done our calculations by considering Coulomb and Yukawa plus exponential potential as interacting barrier for separated fragments and cubic potential for the overlap region, described in section 2 . The results and discussion are given in section 3 . Finally the conclusions are given in section 4 .

\section{Cubic plus Yukawa plus Exponential model:}

In this work, the parent and the emitted cluster are considered to be spheroid, keeping the daughter as spherical. If the emitted cluster has a deformation, say quadrupole deformation only while the daughter nuclear is spherical and if the Q-value of the reaction is taken as the origin, the potential for the post - scission region as the function of the centre of mass distance ' $r$ ' of the fragment is given by

$\mathrm{V}(\mathrm{r})=\mathrm{V}_{\mathrm{c}}(\mathrm{r})+\mathrm{V}_{\mathrm{n}}(\mathrm{r})-\mathrm{V}_{\mathrm{df}}(\mathrm{r})-\mathrm{Q}$

Here $V_{c}$ is the coulomb potential between a spheroid emitted cluster and spherical daughter.

$V_{n}$ is the nuclear interaction energy due to finite range effects of Krappe et al; and $V_{\text {df }}$ is the change in nuclear interaction energy due to quadrupole deformation in the emitted cluster.

National Conference on Current Advancements in Physics $3^{\text {rd }} \& 4^{\text {th }}$ February 2017 84 | Page

Department of Physics, St. John's College, Palayamkottai-627 002, Tamilnadu, India. DOI 10.9790/4861-17002028491 
For a prolate spheroid emitted cluster with longer axis along the fission direction, Pik - Pichak [18] obtained

$$
\mathrm{V}_{\mathrm{c}}(\mathrm{r})=\frac{3}{2} \frac{Z_{d} Z_{e} e^{2} \gamma}{r}\left[\frac{1-\gamma^{2}}{2} \ln \frac{\gamma+1}{\gamma-1}+\gamma\right] ;
$$

and for an oblate spheroid emitted cluster with shorter axis along the fission direction ,

Here,

$$
\mathrm{V}_{\mathrm{c}}(\mathrm{r})=\frac{3}{2} \frac{Z_{d} Z_{e} e^{2}}{r}\left[\gamma\left(1+\gamma^{2}\right) \arctan \gamma^{-1}-\gamma^{2}\right] .
$$

$$
\gamma=\frac{r}{\left(\mathrm{a}_{\mathrm{e}}^{2}-\mathrm{b}_{\mathrm{e}}^{2}\right) 1 / 2}
$$

Where $z_{d}, z_{e}$ are the atomic numbers of the daughter and emitted cluster respectively, $a_{e}$ and $b_{e}$ are the semi major and minor axes of the spheroidal cluster nucleus respectively.

For the overlapping region, we approximate the potential barrier by a third order polynomial in (r) having the form (4)

$$
\begin{gathered}
\mathrm{V}_{\mathrm{c}}(\mathrm{r})=-E_{v}\left[V(r)_{t}+E_{v}\right]\left\{s_{d}\left(\frac{r-r_{i}}{r_{t}-r_{i}}\right)^{2}-s_{e}\left(\frac{r-r_{i}}{r_{t}-r_{i}}\right)^{3}\right\}, \\
\mathrm{r}_{\mathrm{i}} \leq \mathrm{r} \leq \mathrm{r}_{\mathrm{t}}
\end{gathered}
$$

Where

$$
\mathrm{r}_{\mathrm{t}}=\mathrm{a}_{\mathrm{e}}+\mathrm{R}_{\mathrm{d}}
$$

Here $\mathrm{a}_{\mathrm{e}}$ is the semi-major (or) minor axis of the spheroid cluster depending on the prolate (or) oblate shape of the emitted cluster; and $r_{i}$ is the distance between the centers of mass of the daughters and the emitted particle portions in the spheroid parent nucleus. The constants $s_{d}$ and $s_{e}$ appearing in Eq.(4) are determined by requiring that the value of the potential $\mathrm{V}(\mathrm{r})$ and its first derivative be continuous at the contact point $\mathrm{r}=\mathrm{r}_{\mathrm{t}}$. Thus we get

$$
\mathrm{S}_{\mathrm{d}}=3-\mathrm{S} \text { and } \mathrm{S}_{\mathrm{e}}=2-\mathrm{S} \text {; }
$$

Where

$$
\mathrm{S}=\frac{r_{t}-r_{i}}{\left[V\left(r_{t}\right)+E_{v}\right]}\left[V_{c}^{\prime}\left(r_{t}\right)+V_{n}^{\prime}\left(r_{t}\right)-V_{d}^{\prime}\left(r_{t}\right)\right]
$$

If the nuclei have spheroid shape, the radius vector $\mathrm{R}(\theta)$ making an angle $\theta$ with the axis of symmetry locating sharp surface of a deformed nuclei is given by ref [19]

$$
\mathrm{R}(\theta)=\mathrm{R}_{\mathrm{o}}\left[1+\sum_{n=0}^{\alpha} \sum_{m=-n}^{n} \beta_{n m} Y_{n m}(\theta)\right]
$$

Here $\mathrm{R}_{0}$ is the radius of equivalent spherical nucleus.

If we consider spheroid deformation $\beta_{2}$, then

$$
\mathrm{R}(\theta)=R_{o}\left[1+\beta_{2}(5 / 4 \pi)^{1 / 2}\left(\frac{3}{2} \cos ^{2} \theta^{-1 / 2}\right)\right]
$$
becomes

and if the Nilsson's hexadecapole deformation $\beta_{4}$ is also included in the deformation, then Eq. (7)

$$
\mathrm{R}(\theta)=\mathrm{R}_{\mathrm{o}}\left[1+\beta_{2}(5 / 4 \pi)^{1 / 2}\left(3 / 2 \cos ^{2} \theta-1 / 2\right)+\beta_{4}((9 / 4 \pi))^{1 / 2} \frac{1}{8}\left(35 \cos ^{4} \theta-30 \cos ^{2} \theta+3\right)\right]
$$

If hexacontatetrapole deformation $\left(\beta_{6}\right)$ is included in the parent deformation, equation (8) becomes

$$
\begin{aligned}
& \mathrm{R}(\theta)=\mathrm{R}_{\mathrm{o}}\left[1+\beta_{2}(5 / 4 \pi)^{1 / 2}\left(3 / 2 \operatorname{Cos}^{2} \theta-1 / 2\right)+\beta_{4}((9 / 4 \pi))^{1 / 2} \frac{1}{8}\left(35 \cos ^{4} \theta-30 \cos ^{2} \theta+3\right)+\right. \\
& \beta 6134 \pi 116231 \operatorname{Cos} 6 \theta-315 \operatorname{Cos} 4 \theta+105 \operatorname{Cos} 2 \theta-5
\end{aligned}
$$

Expressing the energies in $\mathrm{MeV}$, lengths in fm and time in seconds for calculating the life time of the decay system we use the formula,

$$
\mathrm{T}=\frac{1.433 \times 10^{-21}}{E_{v}}(1+\exp (K))
$$

The zero-point vibration energy $\mathrm{E}_{\mathrm{v}}=\frac{\pi}{2} \frac{\mathrm{h} \sqrt{2 Q / \mu}}{\left(C_{1}+C_{2}\right)}$

Where $\mu$ is the reduced mass of the system and $\mathrm{C}_{1}$ and $\mathrm{C}_{2}$ are the "central" radii of the fragments given by [20]

$$
\mathrm{C}_{\mathrm{i}}=1.18 A_{i}^{1 / 3}-0.48, \quad(i=1,2)
$$

The action integral $\mathrm{K}$ is given by $\mathrm{K}=\mathrm{K}_{\mathrm{L}}+\mathrm{K}_{\mathrm{R}}$

Where $\mathrm{K}_{\mathrm{L}}=\frac{2}{h} \int_{r_{a}}^{r_{t}}\left[2 B_{r} V(r)\right]^{1 / 2} d r$

$$
\mathrm{K}_{\mathrm{R}}=\frac{2}{h} \int_{r_{t}}^{r_{b}}\left[2 B_{r}(r) V(r)\right]^{1 / 2} d r
$$


The limits of integration $r_{a}$ and $r_{b}$ are the two appropriate zeros of the integrand which are found numerically. Q-values for different decay modes are calculated using the experimental binding energies of Audi et al.[21]

\section{Results and discussion:}

In this work, the cluster radioactivity from neutron rich $\mathrm{Bk}$ isotope in the mass region $\mathrm{A}=224$ to 253 have been investigated by using CYE model. It is very important to perform some theoretical calculations in this region which may guide future experiments. The calculated half-lives are in good agreement with the available data. Table1 gives the Logarithmic half-lives for various clusters from neutron rich Bk isotopes including deformation effects. Figures 1-9, represent the comparison of computed logarithmic half lives (with and without deformation) for various clusters with available data. Here we have presented the possible cluster decay which have $T<10^{28} \mathrm{~s}$ and the branching ratio relative to alpha decay $\geq 10^{-18}$. When deformation effects are included, half-life values calculated by inclusion of $\left(\boldsymbol{\beta}_{\mathbf{2 P}}, \boldsymbol{\beta}_{\mathbf{4 P}},\right)$ parameters and $\left(\boldsymbol{\beta}_{\mathbf{2 P}}, \boldsymbol{\beta}_{\mathbf{4 P}}, \boldsymbol{\beta}_{\mathbf{6 P}}\right)$ parameters are found to be decreased, because it reduces the height and width of the barrier. But in the mass region $A=235-258$, the halflife value with inclusion of $\boldsymbol{\beta}_{\mathbf{6}}$ is found to be increased than that of the same calculated without inclusion of $\boldsymbol{\beta}_{6 P}$. This may be due to the structure and internal energy of the Berkelium isotopes. The neutron excess in the parent nuclei also slows down the decay rate. The sign of hexadecapole deformation does not affect the half life time value. The deformation parameter values are taken from the tables of Moller et al[22].The GeigerNuttall plots of various cluster emissions are shown in figure10.The Geiger-Nuttall plots are found to be linear. Our results show that the most probable emitted clusters are alpha like nuclei and the 'daughter nuclei, in which either proton or neutron are equal to magic numbers or around an existing magic numbers which reveals the role of shell structure in exotic decay. Branching ratio relative to alpha decay, $B \cdot R=T_{\text {cluster }} / T_{\alpha}$ is calculated and included in the tabulation.

Table1. Comparison of calculated logarithmic half life of various clusters from Bk isotopes for the case of without and with deformations.

\begin{tabular}{|c|c|c|c|c|c|c|c|c|c|c|c|}
\hline \multirow{3}{*}{$\begin{array}{l}\text { Parent } \\
\text { nuclei }\end{array}$} & \multirow[b]{3}{*}{$\begin{array}{l}\text { Emitte } \\
\mathrm{d} \\
\text { cluster }\end{array}$} & \multirow[b]{3}{*}{$\beta_{2 P}$} & \multirow[b]{3}{*}{$\beta_{4 P}$} & \multirow[b]{3}{*}{$\beta_{6 P}$} & \multirow[b]{3}{*}{$\beta_{2 D}$} & \multirow[b]{3}{*}{$\begin{array}{c}\mathrm{Q} \\
(\mathrm{MeV})\end{array}$} & \multicolumn{4}{|c|}{$\log \mathrm{T}(\mathrm{s})$} & \multirow{3}{*}{ 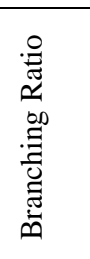 } \\
\hline & & & & & & & \multicolumn{3}{|c|}{ Cal.values } & \multirow{2}{*}{$\begin{array}{c}\text { ASAFM } \\
{[23]}\end{array}$} & \\
\hline & & & & & & & $\begin{array}{l}\text { Without } \\
\text { deforma } \\
\text { tion }\end{array}$ & $\begin{array}{c}\text { with } \\
\left(\beta_{2 P}, \beta_{4 P}\right)\end{array}$ & $\begin{array}{c}\text { with } \\
\left(\beta_{2 P}, \beta_{4 P},\right. \\
\left.\beta_{6 P}\right)\end{array}$ & & \\
\hline \multirow[t]{2}{*}{${ }^{228} \mathrm{Bk}_{97}$} & ${ }^{8} \mathrm{Be}$ & 0.101 & 0.044 & 0.002 & 0.033 & 19.81 & 11.97 & 11.21 & 11.20 & 11.50 & 15.17 \\
\hline & ${ }^{12} \mathrm{C}$ & 0.101 & 0.044 & 0.002 & 0.001 & 35.25 & 15.02 & 14.15 & 14.13 & 14.60 & 18.22 \\
\hline \multirow[t]{2}{*}{${ }^{229} \mathrm{Bk}_{97}$} & ${ }^{8} \mathrm{Be}$ & 0.227 & 0.081 & 0.016 & 0.056 & 18.76 & 14.66 & 12.91 & 12.76 & 12.70 & 17.76 \\
\hline & ${ }^{12} \mathrm{C}$ & 0.227 & 0.081 & 0.016 & 0.001 & 34.61 & 16.18 & 13.82 & 13.63 & 14.10 & 37.71 \\
\hline \multirow{4}{*}{${ }^{230} \mathrm{Bk}_{97}$} & ${ }^{8} \mathrm{Be}$ & 0.227 & 0.081 & 0.008 & 0.089 & 19.07 & 13.79 & 12.13 & 12.05 & 13.20 & 16.69 \\
\hline & ${ }^{12} \mathrm{C}$ & 0.227 & 0.081 & 0.008 & -0.021 & 35.96 & 13.60 & 11.67 & 11.57 & 13.30 & 16.5 \\
\hline & ${ }^{16} \mathrm{O}$ & 0.227 & 0.081 & 0.008 & 0.001 & 52.92 & 14.88 & 12.29 & 12.18 & 14.80 & 17.78 \\
\hline & ${ }^{15} \mathrm{~N}$ & 0.227 & 0.081 & 0.008 & 0.001 & 43.57 & 16.47 & 14.06 & 13.95 & 16.60 & 19.37 \\
\hline \multirow{4}{*}{${ }^{231} \mathrm{Bk}_{97}$} & ${ }^{8} \mathrm{Be}$ & 0.216 & 0.093 & 0.008 & 0.033 & 18.44 & 15.32 & 13.63 & 13.55 & 13.50 & 18.32 \\
\hline & ${ }^{12} \mathrm{C}$ & 0.216 & 0.093 & 0.008 & 0.055 & 35.00 & 15.46 & 13.08 & 12.98 & 13.30 & 18.46 \\
\hline & ${ }^{16} \mathrm{O}$ & 0.216 & 0.093 & 0.008 & 0.001 & 52.27 & 15.82 & 13.03 & 12.92 & 13.90 & 18.82 \\
\hline & ${ }^{15} \mathrm{~N}$ & 0.216 & 0.093 & 0.008 & 0.001 & 43.13 & 17.12 & 14.62 & 14.52 & 15.60 & 20.12 \\
\hline \multirow{3}{*}{${ }^{232} \mathrm{Bk}_{97}$} & ${ }^{8} \mathrm{Be}$ & 0.216 & 0.093 & 0.008 & 0.112 & 17.46 & 18.25 & 16.47 & 16.39 & 17.4 & 19.35 \\
\hline & ${ }^{12} \mathrm{C}$ & 0.216 & 0.093 & 0.008 & 0.089 & 33.36 & 18.48 & 16.19 & 16.09 & 17.8 & 19.58 \\
\hline & ${ }^{16} \mathrm{O}$ & 0.216 & 0.093 & 0.008 & -0.021 & 50.23 & 19.02 & 16.53 & 16.41 & 18.6 & 20.12 \\
\hline \multirow{3}{*}{${ }^{233} \mathrm{Bk}_{97}$} & ${ }^{8} \mathrm{Be}$ & 0.216 & 0.093 & 0.008 & 0.132 & 17.08 & 19.38 & 17.60 & 17.52 & 17.10 & 17.98 \\
\hline & ${ }^{12} \mathrm{C}$ & 0.216 & 0.093 & 0.008 & 0.100 & 32.56 & 20.08 & 17.76 & 17.65 & 17.70 & 20.98 \\
\hline & ${ }^{16} \mathrm{O}$ & 0.216 & 0.093 & 0.008 & 0.001 & 48.92 & 21.15 & 18.13 & 18.01 & 18.80 & 22.05 \\
\hline \multirow{4}{*}{${ }^{234} \mathrm{Bk}_{97}$} & ${ }^{12} \mathrm{C}$ & 0.216 & 0.093 & 0.008 & 0.110 & 30.39 & 24.83 & 22.36 & 22.25 & 23.70 & 19.33 \\
\hline & ${ }^{16} \mathrm{O}$ & 0.216 & 0.093 & 0.008 & 0.078 & 46.25 & 25.84 & 22.82 & 22.69 & 24.90 & 20.34 \\
\hline & ${ }^{26} \mathrm{Mg}$ & 0.216 & 0.093 & 0.008 & -0.073 & 81.10 & 24.47 & 22.94 & 22.78 & 24.90 & 18.97 \\
\hline & ${ }^{30} \mathrm{Si}$ & 0.216 & 0.093 & 0.008 & -0.052 & 97.49 & 24.04 & 22.01 & 21.84 & 24.50 & 18.54 \\
\hline${ }^{235} \mathrm{Bk}_{97}$ & ${ }^{12} \mathrm{C}$ & 0.215 & 0.093 & -0.022 & 0.122 & 31.14 & 23.06 & 20.70 & 20.73 & 20.40 & 22.26 \\
\hline \multirow[t]{2}{*}{${ }^{239} \mathrm{Bk}_{97}$} & ${ }^{30} \mathrm{Si}$ & 0.226 & 0.095 & -0.012 & -0.011 & 96.99 & 24.15 & 20.61 & 20.84 & 23.20 & 20.65 \\
\hline & ${ }^{32} \mathrm{Si}$ & 0.226 & 0.095 & -0.012 & -0.021 & 98.43 & 22.56 & 19.68 & 19.90 & 22.60 & 19.06 \\
\hline${ }^{240} \mathrm{Bk}_{97}$ & ${ }^{32} \mathrm{Si}$ & 0.237 & 0.085 & -0.014 & -0.011 & 98.57 & 22.24 & 19.06 & 19.31 & 23.70 & 17.74 \\
\hline \multirow[b]{2}{*}{${ }^{241} \mathrm{Bk}_{97}$} & ${ }^{32} \mathrm{Si}$ & 0.237 & 0.086 & -0.024 & -0.011 & 98.46 & 22.28 & 19.07 & 19.50 & 22.40 & 18.08 \\
\hline & ${ }^{33} \mathrm{P}$ & 0.237 & 0.086 & -0.024 & 0.001 & 104.21 & 24.85 & 20.78 & 21.25 & 24.20 & 20.65 \\
\hline
\end{tabular}


Role of multipolarity-Six deformation parameter on exotic decay half-lives of Berkelium nucleus

\begin{tabular}{|c|c|c|c|c|c|c|c|c|c|c|c|}
\hline & ${ }^{35} \mathrm{P}$ & 0.237 & 0.086 & -0.024 & 0.001 & 105.13 & 23.95 & 20.23 & 20.67 & 25.50 & 19.75 \\
\hline${ }^{243} \mathrm{Bk}_{97}$ & ${ }^{35} \mathrm{P}$ & 0.237 & 0.073 & -0.027 & 0.001 & 105.31 & 23.43 & 19.96 & 20.45 & 23.70 & 18.53 \\
\hline \multirow{3}{*}{${ }^{245} \mathrm{Bk}_{97}$} & ${ }^{35} \mathrm{P}$ & 0.249 & 0.063 & -0.029 & 0.001 & 101.42 & 28.86 & 25.13 & 25.72 & 28.4 & 19.66 \\
\hline & ${ }^{37} \mathrm{P}$ & 0.249 & 0.249 & 0.249 & 0.249 & 102.84 & 27.29 & 21.57 & 24.17 & 27.7 & 18.09 \\
\hline & ${ }^{38} \mathrm{~S}$ & 0.249 & 0.249 & 0.249 & 0.249 & 109.72 & 27.77 & 21.68 & 24.39 & 27.8 & 18.57 \\
\hline${ }^{247} \mathrm{Bk}_{97}$ & ${ }^{40} \mathrm{~S}$ & 0.249 & 0.051 & -0.032 & 0.001 & 109.05 & 28.99 & 25.39 & 26.02 & 29.4 & 18.09 \\
\hline${ }^{251} \mathrm{Bk}_{97}$ & ${ }^{46} \mathrm{Ar}$ & 0.249 & 0.051 & -0.027 & 0.001 & 123.50 & 28.21 & 23.50 & 25.49 & 29.0 & 17.39 \\
\hline \multirow{6}{*}{${ }^{253} \mathrm{Bk}_{97}$} & ${ }^{49} \mathrm{~K}$ & 0.240 & 0.012 & -0.031 & 0.001 & 129.95 & 28.44 & 25.24 & 25.85 & 29.3 & 15.54 \\
\hline & ${ }^{46} \mathrm{Ar}$ & 0.240 & 0.012 & -0.031 & 0.001 & 122.86 & 28.89 & 25.79 & 26.40 & 29.6 & 15.99 \\
\hline & ${ }^{48} \mathrm{Ar}$ & 0.240 & 0.012 & -0.031 & 0.001 & 123.36 & 28.53 & 25.61 & 26.20 & 29.7 & 15.63 \\
\hline & ${ }^{46} \mathrm{~S}$ & 0.240 & 0.012 & -0.031 & 0.001 & 114.25 & 21.58 & 19.20 & 19.68 & 24.3 & 8.68 \\
\hline & ${ }^{45} \mathrm{Cl}$ & 0.240 & 0.012 & -0.031 & 0.001 & 117.55 & 27.03 & 23.85 & 24.41 & 28.1 & 14.13 \\
\hline & ${ }^{50} \mathrm{Ca}$ & 0.240 & 0.012 & -0.031 & 0.001 & 138.21 & 25.69 & 22.83 & 23.43 & 26.6 & 12.79 \\
\hline \multirow{5}{*}{${ }^{254} \mathrm{Bk}_{97}$} & ${ }^{51} \mathrm{Ca}$ & 0.240 & 0.011 & -0.021 & 0.001 & 137.42 & 26.79 & 23.96 & 24.37 & 29.1 & 9.79 \\
\hline & ${ }^{50} \mathrm{~K}$ & 0.240 & 0.011 & -0.021 & 0.001 & 130.55 & 27.54 & 24.47 & 24.87 & 30.0 & 10.54 \\
\hline & ${ }^{46} \mathrm{Ar}$ & 0.240 & 0.011 & -0.021 & 0.011 & 125.33 & 25.03 & 22.31 & 22.70 & 27.6 & 8.03 \\
\hline & ${ }^{45} \mathrm{Cl}$ & 0.240 & 0.011 & -0.021 & 0.001 & 116.96 & 27.49 & 24.98 & 25.12 & 29.9 & 10.49 \\
\hline & ${ }^{46} \mathrm{Cl}$ & 0.240 & 0.011 & -0.021 & 0.011 & 118.22 & 25.77 & 23.13 & 23.40 & 28.6 & 8.77 \\
\hline \multirow{6}{*}{${ }^{255} \mathrm{Bk}_{97}$} & ${ }^{52} \mathrm{Ca}$ & 0.240 & -0.001 & -0.023 & 0.001 & 137.28 & 26.97 & 24.30 & 24.74 & 28.0 & 11.17 \\
\hline & ${ }^{53} \mathrm{Sc}$ & 0.240 & -0.001 & -0.023 & 0.001 & 141.63 & 29.69 & 26.65 & 27.13 & 29.9 & 13.89 \\
\hline & ${ }^{46} \mathrm{~S}$ & 0.240 & -0.001 & -0.023 & 0.001 & 113.12 & 23.08 & 21.05 & 21.42 & 25.5 & 7.28 \\
\hline & ${ }^{47} \mathrm{~K}$ & 0.240 & -0.001 & -0.023 & 0.001 & 131.52 & 25.50 & 22.94 & 23.38 & 26.4 & 9.7 \\
\hline & ${ }^{46} \mathrm{Ar}$ & 0.240 & -0.001 & -0.023 & 0.011 & 124.88 & 25.56 & 22.99 & 23.42 & 26.8 & 9.76 \\
\hline & ${ }^{47} \mathrm{Ar}$ & 0.240 & -0.001 & -0.023 & 0.011 & 124.16 & 26.82 & 24.22 & 24.65 & 28.0 & 11.02 \\
\hline \multirow{4}{*}{${ }^{256} \mathrm{Bk}_{97}$} & ${ }^{46} \mathrm{~S}$ & 0.229 & -0.004 & -0.013 & 0.001 & 112.53 & 23.90 & 21.91 & 22.13 & 27.4 & 6.2 \\
\hline & ${ }^{48} \mathrm{~K}$ & 0.229 & -0.004 & -0.013 & 0.001 & 131.74 & 25.17 & 22.71 & 22.95 & 27.6 & 7.47 \\
\hline & ${ }^{47} \mathrm{Ar}$ & 0.229 & -0.004 & -0.013 & 0.011 & 124.87 & 25.59 & 23.28 & 23.47 & 28.2 & 7.89 \\
\hline & ${ }^{48} \mathrm{Ar}$ & 0.229 & -0.004 & -0.013 & 0.011 & 124.79 & 25.89 & 23.54 & 23.77 & 28.6 & 8.19 \\
\hline \multirow[b]{2}{*}{${ }^{257} \mathrm{Bk}_{97}$} & ${ }^{46} \mathrm{~S}$ & 0.229 & -0.016 & -0.016 & 0.001 & 113.22 & 22.65 & 20.76 & 21.02 & 25.1 & 4.85 \\
\hline & ${ }^{49} \mathrm{~K}$ & 0.229 & -0.016 & -0.016 & 0.001 & 132.64 & 23.79 & 21.57 & 21.85 & 25.3 & 5.99 \\
\hline \multirow{3}{*}{${ }^{258} \mathrm{Bk}_{97}$} & ${ }^{46} \mathrm{~S}$ & 0.230 & -0.028 & -0.018 & 0.001 & 112.72 & 23.31 & 21.51 & 21.80 & 28.2 & -1.59 \\
\hline & ${ }^{49} \mathrm{~K}$ & 0.230 & -0.028 & -0.018 & 0.011 & 130.34 & 26.24 & 24.97 & 25.31 & 29.4 & 1.34 \\
\hline & ${ }^{50} \mathrm{~K}$ & 0.230 & -0.028 & -0.018 & 0.001 & 131.01 & 27.10 & 24.24 & 24.57 & 28.9 & 2.2 \\
\hline
\end{tabular}

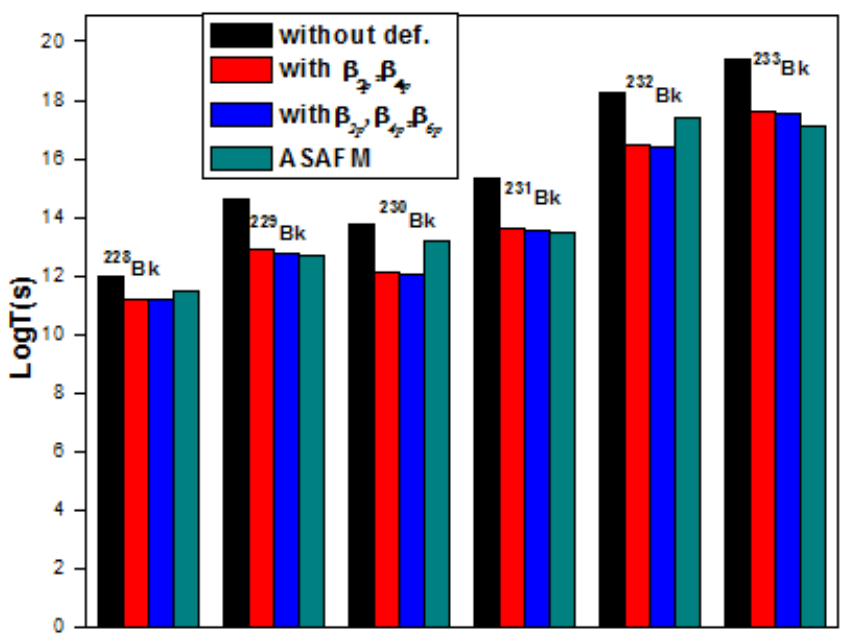

Figure1.Thecomparison of computed ${ }^{8} \mathrm{Be}$ decay half life time with other data. 


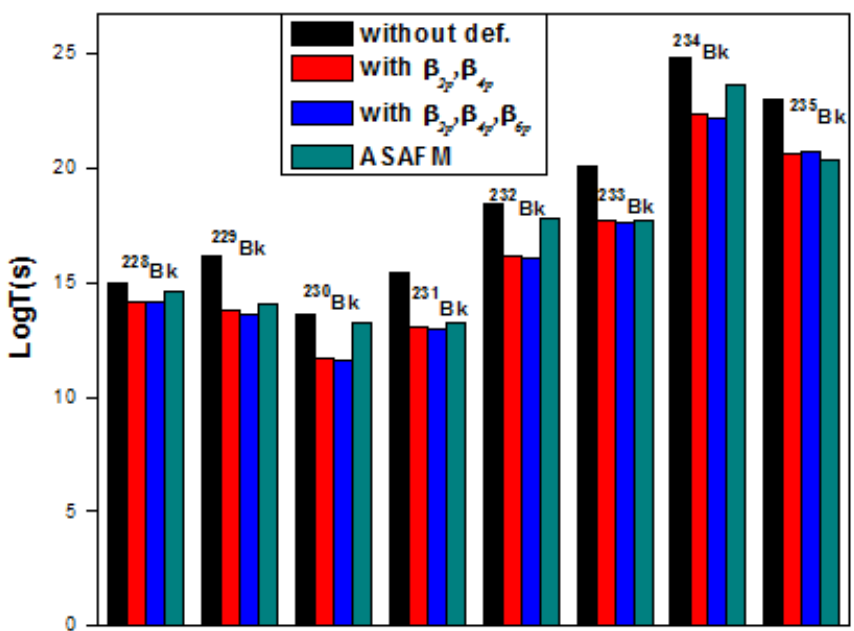

Figure2. The same as for fig. 1 but for ${ }^{12} \mathrm{C}$ decay.

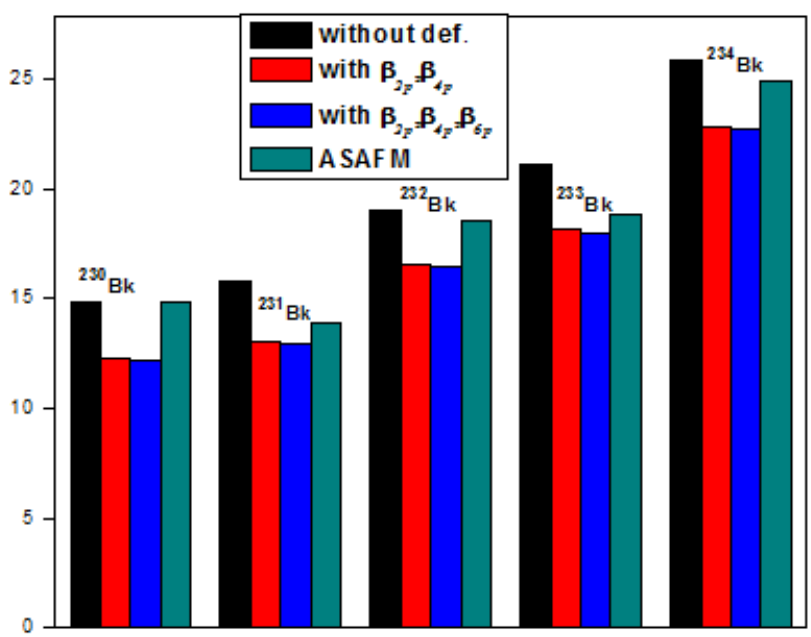

Figure 3. The same as for fig. 1 but for ${ }^{16} \mathrm{O}$ decay.

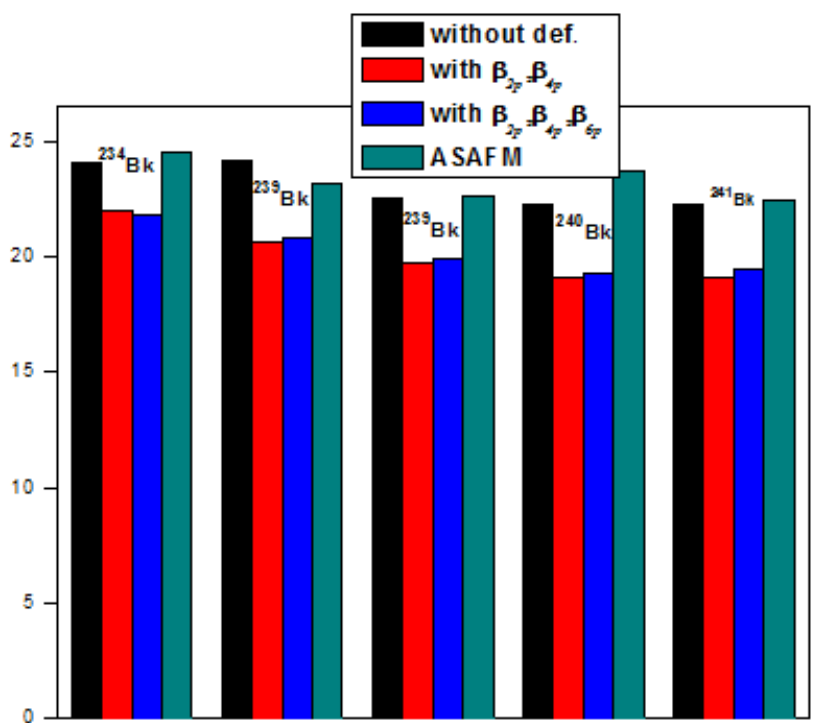

Figure 4. The same as for fig. 1 but for ${ }^{30,32} \mathrm{Si}$ decay. 


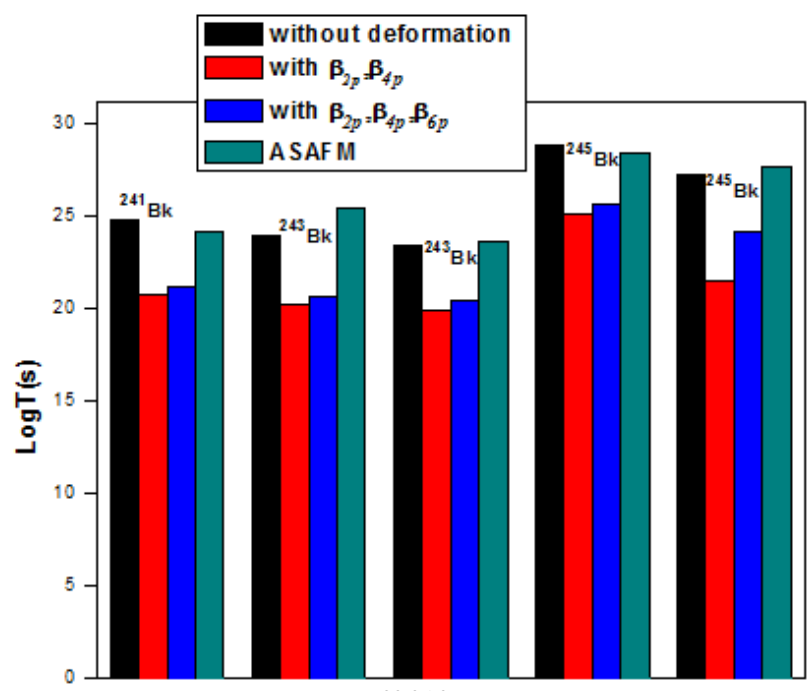

Figure5. The comparisons of computed ${ }^{33,35,37} \mathrm{P}$ decay half life time with other data.

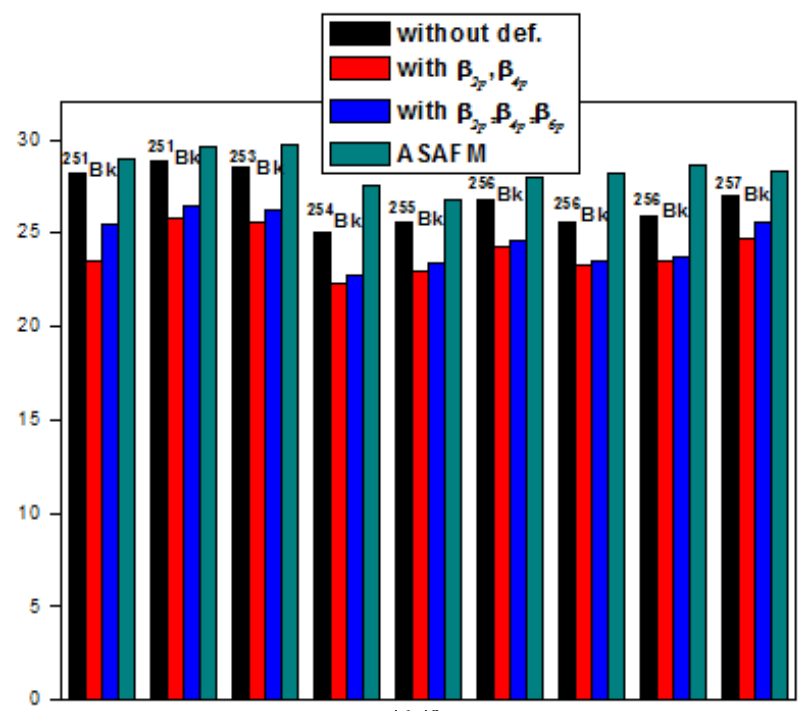

Figure6. The comparisons of computed ${ }^{46-48} \mathrm{Ar}$ decay half life time with other data.

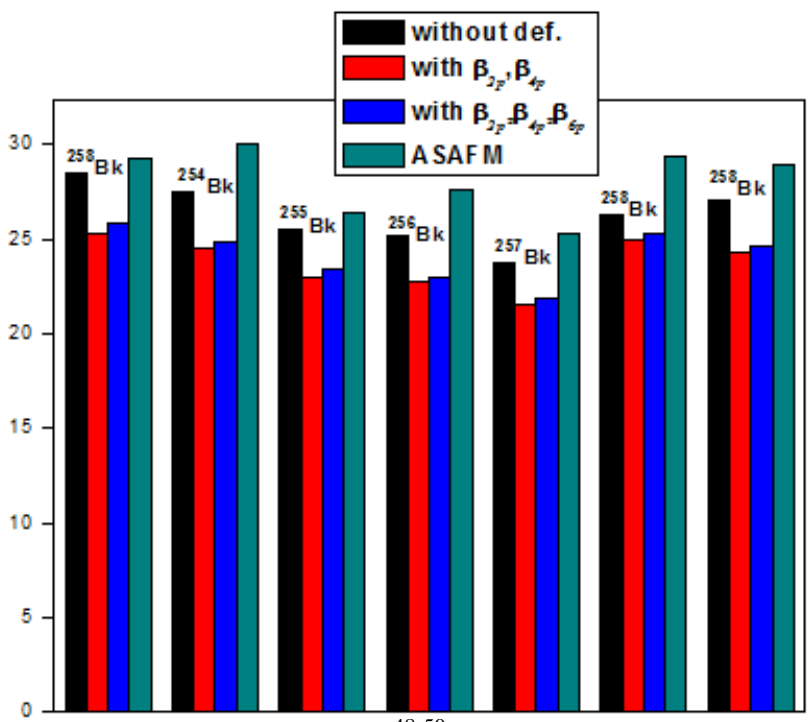

Figure7. The comparisons of computed ${ }^{48-50} \mathrm{~K}$ decay half life time with other data. 


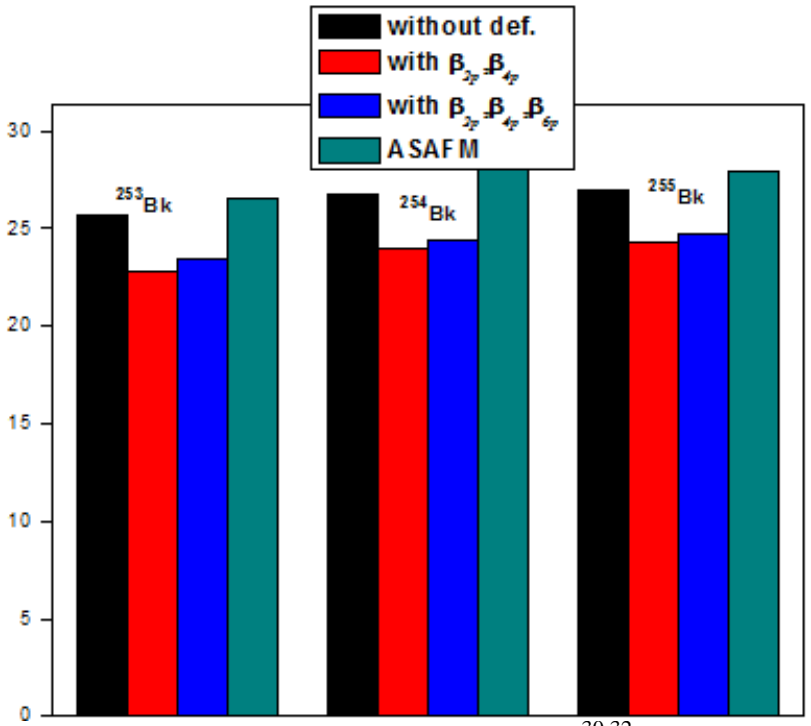

Figure8. The same as for fig.1 but for ${ }^{30,32} \mathrm{Si}$ decay.

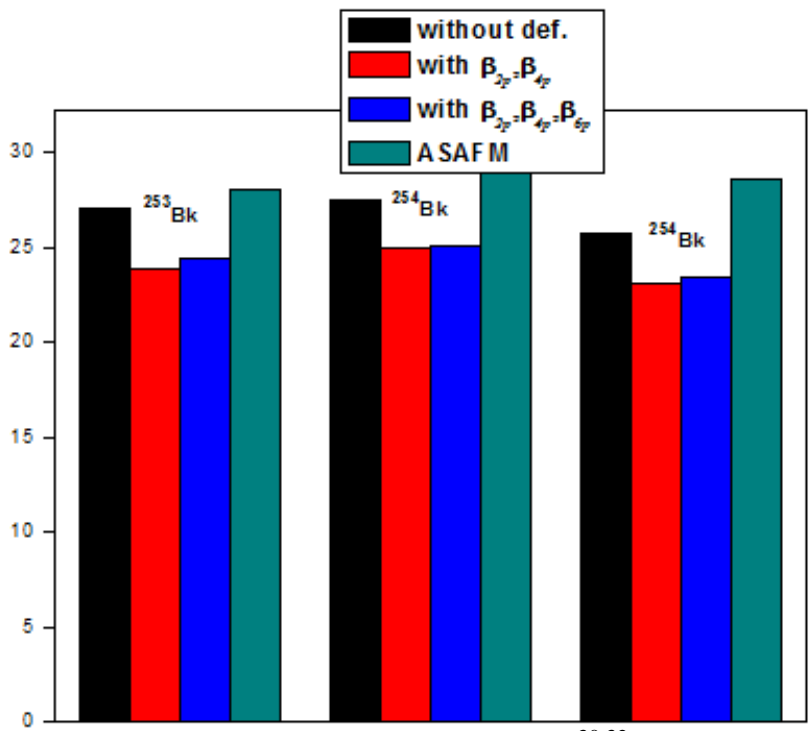

Figure9.The same as for fig.1 but for ${ }^{30,32} \mathrm{Si}$ decay.

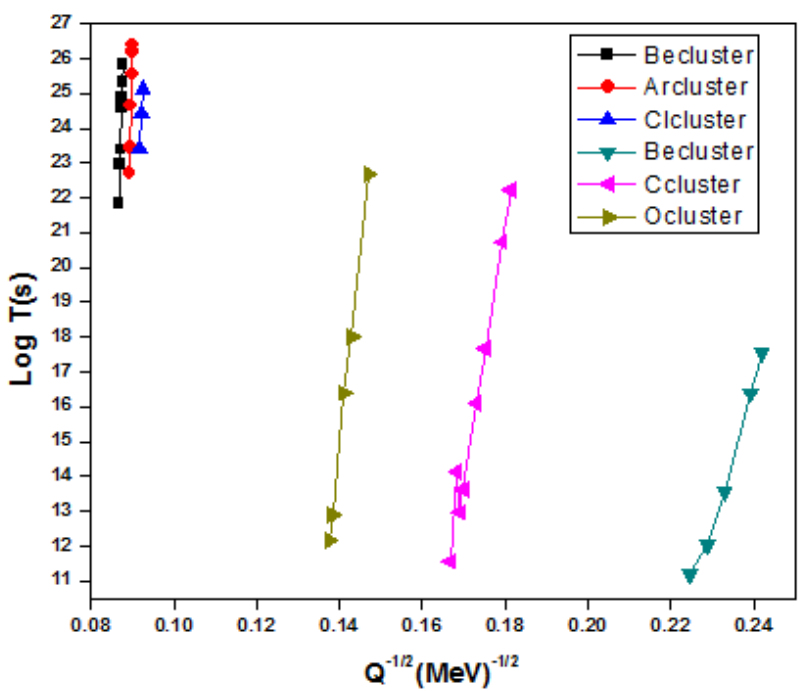

Figure 10.Geiger-Nuttall plots of Berkelium nucleus for various cluster emissions. 


\section{Conclusion:}

We have investigated all the possible decay modes of cluster radioactivity from neutron rich Berkelium nucleus using CYE model. The computed half-life values are compared with the available data. They are good agreement with each other. When deformation effects are included, half-life values are found to be decreased, because it reduces the height and width of the barrier. By incorporating hexacontatetrapole $\left(\beta_{6}\right)$ parameter, the half- life value is found to be increased in the high mass region. This may be due to structure and internal energy of the Berkelium nucleus. Hence the multipolarity six parameter increases the stability of the parent nuclei.

\section{References:}

[1]. A. Sandulescu, D.N.Poenaru and W.Greiner, Fiz.Elen. Chastits At. Yodra 11,1334 (1980); SOV.J.Part Nucl 11,528 (1980).

[2]. H.J.Rose and G.A.Jones, Nature (London) 307, 245 (1984).

[3]. $\quad$ R.Blendowske, T.Fliessbach and H.Walliser,Nucl.Phys.A464,75(1987)

[4]. S.S.Malik and R.K.Gupta, Phys.Rev.C39,1992(1989)

[5]. B.Buck and A.C.Merchant,Phys.Rev.C38,450(1985)

[6]. Y.J.Shi and W.J.Swiatecki, Nucl.Phys.A438,450(1985)

[7]. D.N.Poenaru, M.Ivascu,A.Sandulescu and W.Greiner,Phys.Rev.C32,572(1985)

[8]. K.P.Santhosh, R.K.Biju,S.Sahadevan and A.Joseph 2008 Phys.Scr.77 065210

[9]. R.K.Gupta,M.Horoi,A.Sandulescu,W.Greiner,W.Scheid,J.Phys.G:Nucl.Part.Phys.(1993)2063.

[10]. G.A.Pik Pichak,Sov.J.Nucl.Phys.44(1986)923.

[11]. Y.J.Shi,W.J.Swiatecki, Nucl.Phys.A4649(1987)205.

[12]. G.Shanmugam, G.M.CarmelVigilaBai and B.Kamalaharan, Phys.RevC51,2616(1995).

[13]. Carmel Vigila Bai G.M., ‘ A Systematic Study of Cluster Radioactivity in the Trans-Tin Region', Ph.D., Thesis ,Manmanium Sundaranar University,Tirunelveli-11.

[14]. Carmel Vigila Bai G.M.,and Umai Parvathy J., Proceeding of Department of Atomic Energy (DAE), National Symposium on Nuclear Physics, Volume 60,208(2015).

[15]. Carmel Vigila Bai G.M.,and Nithya Agnes R., 'Alpha decay and cluster decay of some neutron rich actinide nuclei' communicated and accepted to pramana.

[16]. Carmel Vigila Bai G.M.,and Nithya Agnes R., Proceeding of Department of Atomic Energy (DAE), National Symposium on Nuclear Physics, Volume 60,208(2015)

[17]. Carmel Vigila Bai G.M.,and Nithya Agnes R.,Proceeding of Department of Atomic Energy (DAE), National Symposium on Nuclear Physics, Volume 61,208(2016).

[18]. G.A. Pik - Pichakt Sovt. J. Nucl. Phys. 44,923 (1980).

[19]. H.J.Krappe, JR Nix and AJ. Sierk, Phys Rev C 20,992 (1979).

[20]. R.Hofstadeter, in Nuclear Radii, Vol. 2 of Nuclear Physics and Technology, edited by H.Schopper(Springer-Verlag, Berlin,1967)

[21]. G.Audi,A.H.Wapstra,C.Thivault,Nucl.Phys. A 729 (2003)337.

[22]. P. Möller, A.J. Sierk, T. Ichikawa, H. Sagawa Nuclear ground-state masses and deformations: FRDM(2012)

[23]. D .N.Poenaru ,D.Schnabel, and W.Greiner, Atomic Data and Nuclear Data Tables 48, 231 - 327 (1991) 